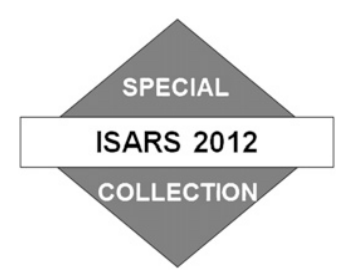

\title{
Lidar-Observed Stress Vectors and Veer in the Atmospheric Boundary Layer
}

\author{
JACOB BERG AND JAKOB MANN \\ Technical University of Denmark, Risø Campus, Roskilde, Denmark \\ EDWARD G. PATTON \\ National Center for Atmospheric Research,* Boulder, Colorado
}

(Manuscript received 29 November 2012, in final form 21 March 2013)

\begin{abstract}
This study demonstrates that a pulsed wind lidar is a reliable instrument for measuring angles between horizontal vectors of significance in the atmospheric boundary layer. Three different angles are considered: the wind turning, the angle between the stress vector and the mean wind direction, and the angle between the stress vector and the vertical gradient of the mean velocity vector. The latter is assumed to be zero by the often applied turbulent-viscosity hypothesis, so that the stress vector can be described through the vertical gradient of velocity. In the atmospheric surface layer, where the Coriolis force is negligible, this is supposedly a good approximation. High-resolution large-eddy simulation data show that this is indeed the case even beyond the surface layer. In contrast, through analysis of WindCube lidar measurements supported by sonic measurements, the study shows that it is only valid very close to the surface. The deviation may be significant even at $100 \mathrm{~m}$. This behavior is attributed to mesoscale effects.
\end{abstract}

\section{Introduction}

Wind lidars have significantly matured over the last few decades (Emeis et al. 2007). Their strength for measuring the mean vertical wind profile up to hundreds of meters is obvious (Peña et al. 2009, 2010). Measuring turbulent second-order statistics with wind lidars has been done extensively (Gal-Chen et al. 1992; Frehlich and Cornman 2002; Lothon et al. 2009; Pichugina et al. 2008) and careful comparisons with mast-mounted sonic anemometers have been carried out by Sathe et al. (2011), who modeled the biases in the second-order statistics because of the averaging volume and the combination of line-of-sight velocities at different positions. The biases on the variances of the three velocity components were shown to vary significantly with stability, reflecting varying

\footnotetext{
* The National Center for Atmospheric Research is sponsored by the National Science Foundation.

Corresponding author address: Jacob Berg, Department of Wind Energy, Technical University of Denmark, Ris $\varnothing$ Campus, Frederiksborgvej 399, 4000 Roskilde, Denmark.

E-mail: jbej@dtu.dk
}

turbulence length scales. Mann et al. (2010) showed that by using the two-beam method proposed by Eberhard et al. (1989), the WindCube pulsed lidar can measure turbulent stress in the mean wind direction in fair agreement with sonic anemometer measurements. Other investigations of the capabilities of lidars have used profilers for the validation (Mayor et al. 1997).

In this contribution we will focus on the ability of a wind lidar to measure angles between horizontal vectors of significance in the atmospheric boundary layer (ABL). In principle, a conical scanning wind lidar should be able to measure even very small systematic angles that are difficult to measure by both wind vanes and sonic anemometers owing to the manual mounting on booms.

We will confine our investigation to the following three angles: 1) the turning of the wind relative to the wind direction at some fixed height, 2) the angle between the horizontal mean wind vector and the stress vector, and 3) the angle between the vertical gradient of the horizontal velocity vector and the stress vector.

In the surface layer, all three angles are assumed to be zero following standard Monin-Obukhov similarity theory because of the relatively weak influence of the Coriolis force here. In addition, the streamwise vertical 
momentum flux is controlled by wind shear in agreement with the turbulent-viscosity hypothesis (Pope 2000):

$$
\left\langle u^{\prime} w^{\prime}\right\rangle=-\nu_{T} \frac{d U}{d z}
$$

where the turbulent eddy viscosity is given by $\nu_{T}$. We have used the normal micrometeorological coordinate system convention that $x$ is aligned with the near-surface mean wind direction - that is, $U(z)$ is the mean wind with superimposed fluctuations $u^{\prime}(x, y, z, t)$, and lateral and vertical fluctuations $v^{\prime}(x, y, z, t)$ and $w^{\prime}(x, y, z, t)$, respectively, forming a right-handed coordinate system. Terms in angle brackets denote the ensemble mean here, taken as time average using ergodicity. We have used 30-min averages of the atmospheric measurements throughout the paper.

Our main objective is to study the angles $\alpha$ and $\beta$, which we define as

$$
\alpha=\tan _{2}^{-1}(U, V)-\tan _{2}^{-1}\left(-\left\langle u^{\prime} w^{\prime}\right\rangle,-\left\langle v^{\prime} w^{\prime}\right\rangle\right) \text { and }
$$

$\beta=\tan _{2}^{-1}\left(\frac{d U}{d z}, \frac{d V}{d z}\right)-\tan _{2}^{-1}\left(-\left\langle u^{\prime} w^{\prime}\right\rangle,-\left\langle v^{\prime} v^{\prime}\right\rangle\right)$

respectively, where the notation $\tan _{2}^{-1}(x, y)$ translates into the angle, $\tan ^{-1}(y / x)$, considering the quadrant to which the point $(x, y)$ belongs. Therefore, the angle between the positive $x$ axis and the vector defined by $(x, y)$ is positive for counterclockwise angles.

The stress vector $\boldsymbol{\tau}$ is defined as

$$
\boldsymbol{\tau}=-\rho\left(\left\langle u^{\prime} w^{\prime}\right\rangle,\left\langle v^{\prime} w^{\prime}\right\rangle\right),
$$

where $\rho$ is the air density. The stress vector is in the opposite direction to the mean momentum flux vector, and the above-mentioned definitions give positive angles for situations where the stress vector is oriented to the right of the mean wind vector (in the case of $\alpha$ ) and of the vertical gradient of the mean wind vector (in the case of $\beta$ ), respectively (see the sketch in Fig. 1).

Thus, close to the surface in homogeneous conditions where, by definition, $V=0$ and $\left\langle v^{\prime} w^{\prime}\right\rangle=0$, we obtain the well-known result $\alpha=0$ and $\beta=0$. Whereas $\beta$ has not received much attention in the literature, besides the fact that Eq. (1) is always assumed to be valid in the surface layer, $\alpha$ has been the focus in a number of studies: Grachev et al. (2003) discussed a nonzerovalued $\alpha$ observed in offshore conditions and attributed this to ocean swell. In the studies reported by Barnardes and Dias (2010) and Weber (1999), the scatter around the zero angle $\alpha$ is discussed in terms of averaging and

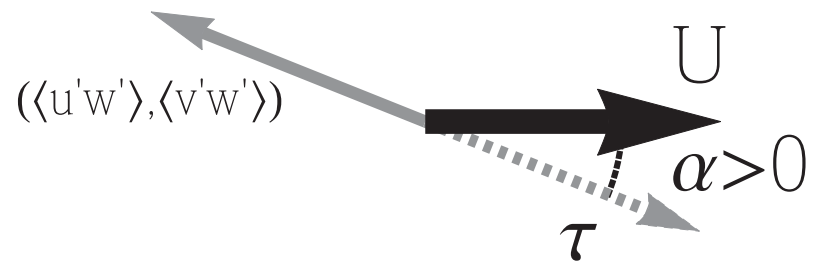

FIG. 1. Orientation of the angle $\alpha$, where $\mathbf{U}=(U, V)$ is the horizontal mean wind speed vector and $\tau=-\rho\left(\left\langle u^{\prime} w^{\prime}\right\rangle,\left\langle v^{\prime} w^{\prime}\right\rangle\right)$ is the stress vector.

inhomogeneous surface conditions. There is no focus on the fact that perhaps $\alpha$ being nonzero even close to the surface is due to the Coriolis force. For a thorough discussion on the angle $\alpha$ and the Coriolis force, we refer to Wyngaard (2010, part II).

According to the above discussion, the notion, close to the surface becomes a central part of this study, and a most relevant question seems to be, How far up do we really have to go before the Coriolis force becomes important and violates the zero angle ansatz for $\alpha$ ?

\section{Bolund sonic measurements}

The motivation for this study was the Bolund measurement campaign held in Denmark from December 2007 to February 2008 (Berg et al. 2011). Measurements indicated that the surface fluxes (sonic anemometer, Metek USA-1, measurements at 5 and $12 \mathrm{~m}$ ) were misaligned with the mean wind, that is, $\alpha \neq 0$. Figures $2 \mathrm{a}, \mathrm{c}$ show histograms of $\alpha$ for upstream conditions with fetch lengths of 0.8 and $7 \mathrm{~km}$, respectively (distance from measurement point to nearest land site in the upstream wind direction). The measurements $(12 \mathrm{~m}$ above sea level) were performed on an offshore platform in a shallow-water fjord and should therefore not be influenced by swell as discussed in Grachev et al. (2003).

When calculating mean angles, such as $\langle\alpha\rangle$, we utilize the trigonometric average

$$
\langle\alpha\rangle=\tan ^{-1}\left(\frac{s_{\alpha}}{c_{\alpha}}\right),
$$

where $s_{\alpha}=\langle\sin \alpha\rangle$ and $c_{\alpha}=\langle\cos \alpha\rangle$. This method gives an equal weight to all angles independent of the magnitude of either the stress vector or the wind vector. The standard deviation is calculated with the method by Yamartino (1984), which by Weber (1997) was shown to be superior compared to a large number of methods:

$$
\sigma_{\alpha}=\sin ^{-1} \epsilon\left[1+\left(\frac{2}{\sqrt{3}}-1\right) \epsilon^{3}\right],
$$

where $\epsilon^{2}=1-\left(s_{\alpha}+c_{\alpha}\right)$. 

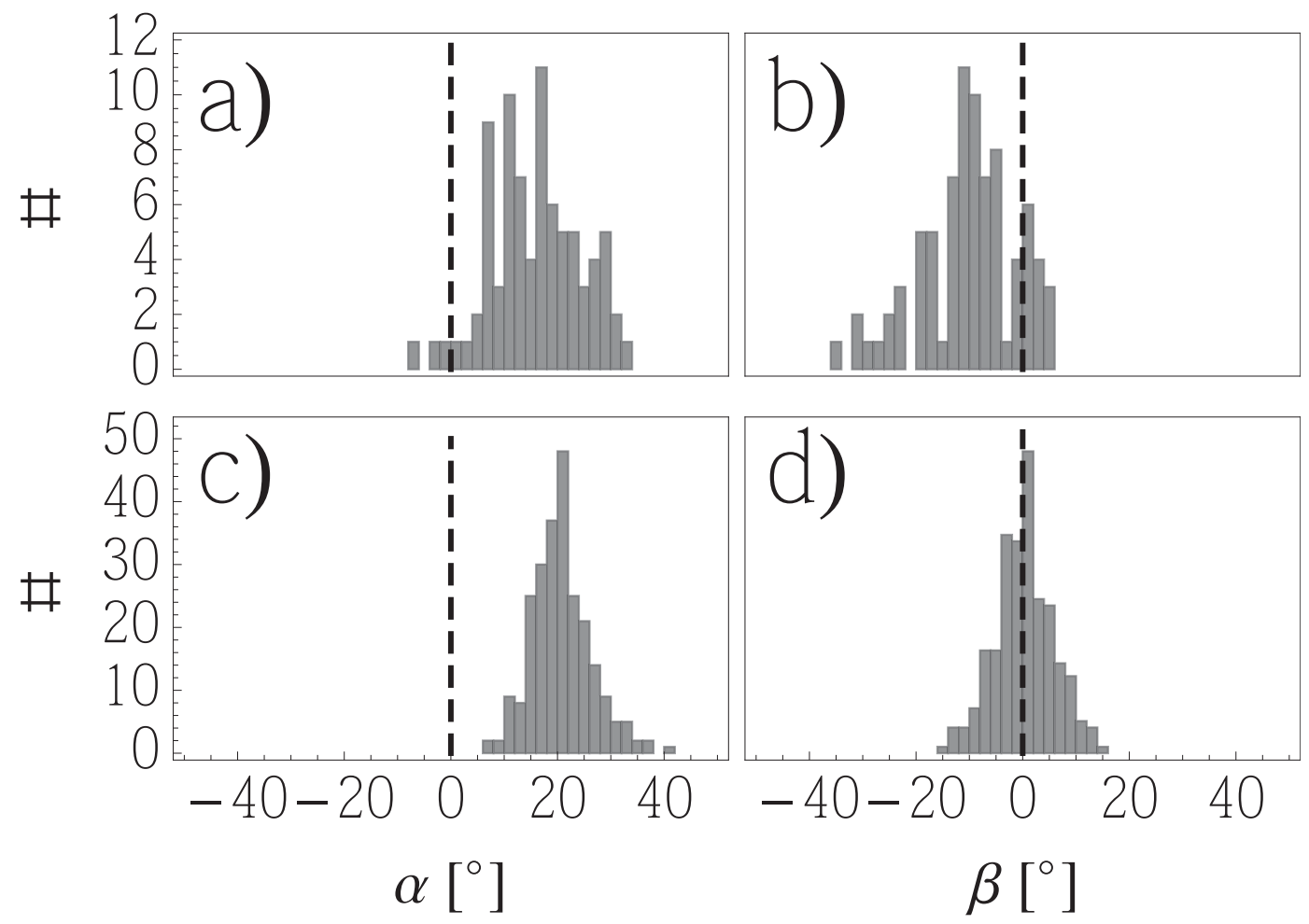

FIG. 2. Histogram of the sonic-measured angles at Bolund: (a) $\alpha$ and (b) $\beta$ measured for short fetch ( $800 \mathrm{~m}$ ), and (c) $\alpha$ and (d) $\beta$ measured for long fetch $(7 \mathrm{~km})$. For the short fetch, a total of 162 samples of 30-min averages of momentum flux and wind speed were used. For the long fetch, 245 samples of 30-min averages were used. Only neutral conditions are considered.

The mean values for the two fetch lengths 0.8 and $7 \mathrm{~km}$ of $\alpha$ are $15^{\circ}$ and $21^{\circ}$, respectively. However, the standard deviation is larger for the shorter fetch $\left(8.5^{\circ}\right.$ versus $\left.5.5^{\circ}\right)$, as expected, because of inhomogeneous conditions and accompanying high-turbulence levels.

The sign of $\alpha$ is in agreement with an Ekman spiral in the Northern Hemisphere (i.e., the wind vector is to the left of the stress vector), although the values are much higher than our expectations of $\alpha=\beta=0$ considering the low measurement height $(12 \mathrm{~m})$.

We have also calculated the angle, $\beta$, from the Bolund data, where the gradient wind speed vector was calculated as a finite difference between the two heights, 5 and $12 \mathrm{~m}$. The result is presented in Figs. $2 \mathrm{~b}$ and $2 \mathrm{~d}$. For the short fetch, the mean of $\beta$ is equal to $-10^{\circ}$, while it is zero for the long fetch as assumed by Eq. (1). Again, the variance was highest in the short fetch case $\left(9.0^{\circ}\right.$ versus $5.5^{\circ}$ ).

Thus, Fig. 2 indicates that the Coriolis force is important even very close to the surface $(5-12 \mathrm{~m})$. As already mentioned the fixed coordinate system of a conically scanning wind lidar makes it a suitable instrument for measuring angles between horizontal vectors of different atmospheric quantities. The remainder of this paper is therefore devoted to measurements performed using the WindCube pulsed wind lidar.

\section{Lidar versus sonic measurements}

We used a conically scanning pulsed wind lidar WindCube WLS7 manufactured by the French company Leosphere (Mann et al. 2009). Besides the successful measurements of turbulent fluxes in the streamwise direction mentioned in the introduction, Mann et al. (2010) also found that for heights above approximately $50 \mathrm{~m}$, there is a constant systematic error when measuring $\left\langle u^{\prime} w^{\prime}\right\rangle$ of approximately $20 \%$. In the data analysis performed in this manuscript, the transverse component $\left\langle v^{\prime} w^{\prime}\right\rangle$ is of the same order of magnitude as the streamwise component $\left\langle u^{\prime} w^{\prime}\right\rangle$. We used the same WindCube as that in Mann et al. (2010) for estimating both the streamwise and the transverse component of momentum flux from the two-beam method.

The two-beam method originally proposed by Eberhard et al. (1989) considers a laser beam pointing in the direction

$$
\mathbf{n}(\theta)=(\sin \phi \cos \theta, \sin \phi \sin \theta, \cos \phi),
$$


where $\phi \sim 27^{\circ}$ is the half-opening angle of the conical scan and $\theta$ is the horizontal orientation relative to some fixed direction. If $v\left[d_{f} \mathbf{n}(\theta)\right]$ denote the wind velocity at the range gate centered at a distance $d_{f}$ from the lidar, then the measured velocity along the beam is given by

$$
v_{r}(\theta)=\mathbf{n}(\theta) \cdot v\left[d_{f} \mathbf{n}(\theta)\right]
$$

The variance of the laser beam, calculated from the time series of $v_{r}(\theta)$, is then given by

$$
\begin{aligned}
\sigma^{2}\left[v_{r}(\theta)\right]= & \left\langle\left[v_{r}^{\prime}(\theta)\right]^{2}\right\rangle=\sigma_{u}^{2} \sin ^{2} \phi \cos ^{2} \theta+\sigma_{v}^{2} \sin ^{2} \phi \sin ^{2} \theta+\sigma_{w}^{2} \cos ^{2} \phi+2\left\langle u^{\prime} v^{\prime}\right\rangle \sin ^{2} \phi \cos \theta \sin \theta \\
& +2\left\langle u^{\prime} w^{\prime}\right\rangle \cos \phi \sin \phi \cos \theta+2\left\langle v^{\prime} w^{\prime}\right\rangle \cos \phi \sin \phi \sin \theta
\end{aligned}
$$

The four beams are positioned at the azimuthal angles, $\theta=\left\{0^{\circ}, 90^{\circ}, 180^{\circ}, 270^{\circ}\right\}$. We denote $\Theta$ as the mean wind direction and define

$$
\begin{aligned}
\Delta_{0} & =\sigma^{2}\left[v_{r}(\Theta)\right]-\sigma^{2}\left[v_{r}\left(\Theta+180^{\circ}\right)\right] \text { and } \\
\Delta_{180} & =\sigma^{2}\left[v_{r}\left(\Theta+90^{\circ}\right)\right]-\sigma^{2}\left[v_{r}\left(\Theta+270^{\circ}\right)\right] .
\end{aligned}
$$

The momentum flux is then given by

$$
\left(\begin{array}{c}
\left\langle u^{\prime} w^{\prime}\right\rangle \\
\left\langle v^{\prime} w^{\prime}\right\rangle
\end{array}\right)=\frac{1}{2 \sin 2 \phi}\left(\begin{array}{cc}
\cos \Theta & -\sin \Theta \\
\sin \Theta & \cos \Theta
\end{array}\right)\left(\begin{array}{c}
\Delta_{0} \\
\Delta_{180}
\end{array}\right)
$$

that is, two beams are needed for each of the two horizontal flux components.

The site was the Danish test site for large wind turbines at Høvsøre in Denmark. We considered easterlies for which the upstream surface conditions are close to homogeneous, although with some patches of forest and urbanized areas within $10 \mathrm{~km}$ (see Fig. 3). Because of the constant pulse length of the lidar, the length along the beam over which data were averaged was constant with respect to distance from ground. This distance is associated with the vertical resolution and is approximately $30 \mathrm{~m}$. With a half-opening angle of $27^{\circ}$, the horizontal distance increases from $35 \mathrm{~m}$ at a height of $40 \mathrm{~m}$ to $180 \mathrm{~m}$ at a height of $200 \mathrm{~m}$. The latter is important for the validity of the assumption of homogeneous terrain when analyzing the data.

In Fig. 4 we present simultaneous measurements from the WindCube and sonic anemometers (mounted on the 116-m meteorological tower positioned within a distance of $25 \mathrm{~m}$ to the WindCube) of the two components of the stress vector (left panels) and the angle, $\alpha$ (right panels).

We present data in three different stability classes:

$$
\begin{array}{ll}
\text { Stable: } & 10 \leq L \leq 500, \\
\text { Neutral: } & 500<L \text { or } L<-1500, \text { and } \\
\text { Unstable: } & -1500 \leq L \leq-10,
\end{array}
$$

where $L$ is the Obukhov length, defined as

$$
L=-\frac{T_{0}}{\kappa g} \frac{u_{\star}^{3}}{Q_{0}},
$$

with the surface temperature $T_{0}$, the surface heat flux $Q_{0}=\left\langle w^{\prime} \theta^{\prime}\right\rangle$, and the friction velocity $u_{\star}=\sqrt{-\left\langle u^{\prime} w^{\prime}\right\rangle}$ measured by a sonic anemometer at $20 \mathrm{~m}$ mounted on the meteorological tower. Also, the acceleration due to gravity $g$ is $9.8 \mathrm{~m} \mathrm{~s}^{-2}$, and the von Kármán constant $\kappa$ is 0.4 .

When the individual components are considered, we find much lower correlation between the lidar and sonic anemometers for $\left\langle u^{\prime} w^{\prime}\right\rangle$ (red) than that for $\left\langle u^{\prime} w^{\prime}\right\rangle$ (blue). This could be due to the smaller length scale for the transverse component. In Sathe and Mann (2012) it is shown how a wind lidar spectrum for the individual velocity components are truncated for high wavenumbers in a nontrivial way. We believe the cross variance spectrum shows similar behavior.

We expect the correlation between lidar and sonic measurements to be smallest in stable conditions because of the smaller turbulent scales. Stable conditions also have enhanced wind shear. This effect would, on the other hand, only contribute to larger scatter when averaged over the lidar gate length. We observe that this is indeed the case. The agreement is best in the unstable case. In all cases, however, the coefficient of determination $R^{2}$ is very low, indicating large scatter. In a recent study by Bradley (2013), it is shown that more than two-thirds of the reduction in $R^{2}$ between a mast-mounted "point" instrument (like a sonic anemometer) and a sodar when comparing wind speeds is due to the decorrelated wind speeds measured by the two instruments rather than just sodar error. We believe that this should also be the case for a comparison between a lidar and a sonic anemometer.

At Høvsøre we also observe a large offset in $\alpha$ between the lidar and sonic measurements. In Table 1 we present the mean values of $\alpha$ for three different heights ( $80 \mathrm{~m}$ was presented in Fig. 4). The offset between lidar and sonic measurements observed in Fig. 4 is evident as 


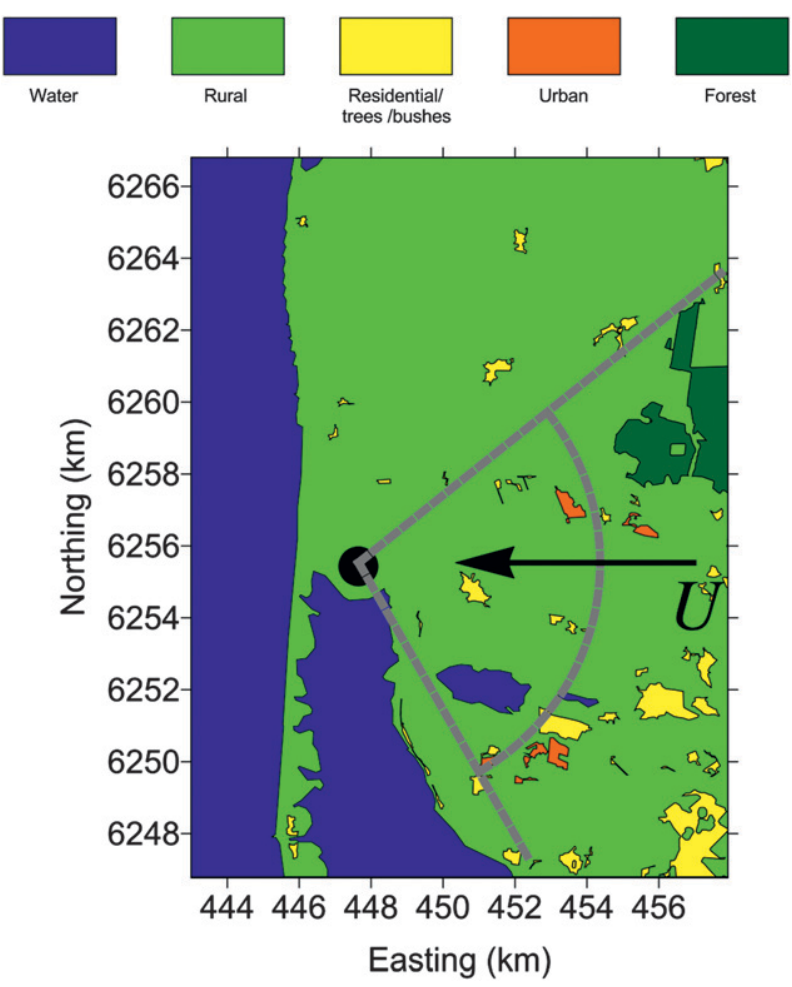

FIG. 3. Map of upstream surface terrain (indicated as the area between the two dashed lines at $50^{\circ}$ and $150^{\circ}$ ) at the Høvsøre measurement site in Denmark. Coordinates are given from Universal Transverse Mercator zone 32 (UTM32). (Original figure produced by Sven-Erik Grynning.)

a large difference in the mean values of the angles measured by the two instruments. Worth noting is the standard error of the mean, which is of similar size between the two instruments. In general the sonic anemometer measures much larger values than the lidar.

We used the rapid distortion theory (RDT) turbulence model by Mann (1994) in order to investigate the difference in correlation between the two momentum flux components. Since the model lacks Coriolis force, two things were done: 1) we checked whether the two components of momentum flux would be biased if one of the laser beams were not aligned with the mean wind direction; and 2) we rotated the spectral tensor off the mean wind direction, so that the momentum flux was not aligned with the mean wind direction. In both cases a deviation of no more than $1^{\circ}$ was found. Therefore, this cannot explain the observed poor lidar-sonic correlation of the transverse component $\left\langle v^{\prime} w^{\prime}\right\rangle$ compared to that of the longitudinal component $\left\langle u^{\prime} w^{\prime}\right\rangle$. We used standard neutral ABL parameters when running the RDT model.

From Fig. 4 we conclude that although the uncertainty of $\alpha$ is large, its sign (positive) is found both from the sonic anemometer and the lidar (the vast majority of points lie in the first quadrant).

\section{Vertical profiles}

This paper's main result is the vertical profiles of the angles $\alpha$ and $\beta$. The most obvious way to calculate these is by the trigonometric average in Eq. (5). As mentioned before, this method gives an equal weight to all angles independent of the magnitude of either the stress vector or the wind vector.

In Fig. 5 we plot $\alpha$ and $\beta$ in neutral conditions with the $40-\mathrm{m}$ wind speed being between 8 and $10 \mathrm{~m} \mathrm{~s}^{-1}$. The peak-to-peak variation is approximately $40^{\circ}$ and $60^{\circ}$ for $\alpha$ and $\beta$, respectively. In Fig $5 \mathrm{a},\langle\alpha\rangle$ is positive and increases with height as expected because of the influence of the Coriolis force. In Fig. 5b, $\langle\beta\rangle$ is slightly negative and decreases with height. The standard error of the mean $\sigma / \sqrt{N}$ is also shown and indicates that the signs of $\alpha$ and $\beta$ are statistical significant. The individual profiles of both $\alpha$ and $\beta$ are often nonmonotonic-most often for $\beta$. This indicates that the profiles, especially those of the vertical gradient of the velocity vector, are not that well determined over runs of only $30 \mathrm{~min}$. Since longer time series are more vulnerable to nonstationary effects, such as the daily cycle, we suggest another method for obtaining the angles-a method that is not contaminated by the uncertainty of the individual runs.

In the new approach, we averaged the different components of the momentum vector, the 30-min mean wind vector, and the vertical gradient of the 30-min mean wind vector, and then took the angle of the resulting averaged vectors. Doing this, we effectively gave more weight to situations in which the momentum flux was large. The results are presented in Fig. 6 .

In addition to $\alpha$ and $\beta$, we also show the angle $\gamma$, which is the veer angle-that is, the turning of the horizontal mean wind vector relative to the wind direction at $40 \mathrm{~m}$ :

$$
\gamma=\tan _{2}^{-1}(U, V)_{z=40 \mathrm{~m}}-\tan _{2}^{-1}(U, V) .
$$

\section{a. Vertical profiles of $\gamma$}

Considering $\gamma$ in Fig. 6 (green curves), we see that the wind turns most with height in stable conditions (top panel) and least in unstable conditions (bottom panel). If we consider the dependence of wind speed, then we see that for lower wind speeds, the magnitude of the turning increases. This is all in agreement with common knowledge: when the wind is lower, the height of the boundary layer is typically smaller and a larger variation of $\gamma$ is expected (this is also true for $\alpha$ and $\beta$ ). An average 

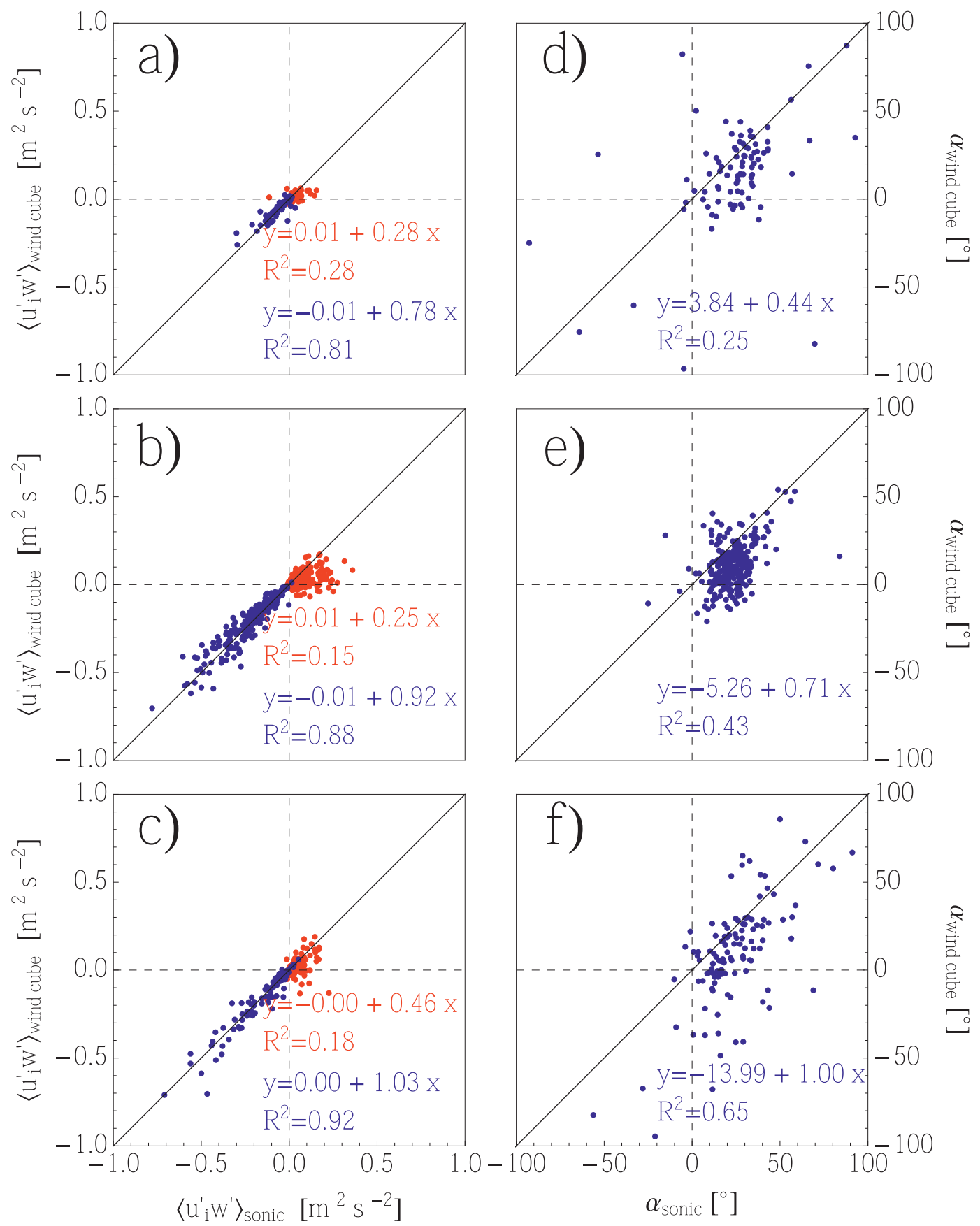

FIG. 4. Sonic anemometer vs WindCube at $80 \mathrm{~m}$. Measurements of $\left\langle u^{\prime} w^{\prime}\right\rangle$ (blue) and $\left\langle v^{\prime} w^{\prime}\right\rangle$ (red) in (a) stable, (b) neutral, and (c) unstable conditions using 101, 263, and 114 samples of 30-min averages, respectively. Measurements of $\alpha$ in (d) stable, (e) neutral, and (f) unstable conditions. Linear regression coefficient and $R^{2}$ are denoted in each panel.

angle of approximately $20^{\circ}$ is observed in the most extreme situation of low winds under stable stratification. Since we do not have lidar measurements either below 40 or above $200 \mathrm{~m}$, we could not capture the full Ekman spiral and hence could not estimate in which way the observed turning actually scales with the surface and the top of the boundary layer parameters (Clarke and Hess 1974).

\section{b. Vertical profiles of $\alpha$}

Vertical profiles of $\alpha$ are presented by the red curves in Fig. 6. Again, the largest angles are observed in the 
TABLE 1. Statistics of $\alpha$ at three heights $(40,80$, and $100 \mathrm{~m})$, where $\langle\alpha\rangle^{l}$ and $\langle\alpha\rangle^{s}$ denote the means for lidar and sonic measurements, respectively. Error is calculated as the standard error of the mean $\sigma_{\alpha} / \sqrt{N}$, where $N$ is the total number of 30-min averages used. For the stable, neutral, and unstable classes, $N$ is 101, 263, and 114, respectively.

\begin{tabular}{lrrr}
\hline & \multicolumn{1}{c}{ Stable } & \multicolumn{1}{c}{ Neutral } & Unstable \\
\hline$\langle\alpha\rangle_{40}^{l}\left({ }^{\circ}\right)$ & $12.0 \pm 2.2$ & $7.3 \pm 0.7$ & $6.5 \pm 2.2$ \\
$\langle\alpha\rangle_{40}^{s}\left({ }^{\circ}\right)$ & $3.3 \pm 2.6$ & $8.4 \pm 0.9$ & $5.0 \pm 2.3$ \\
$\langle\alpha\rangle_{80}^{l}\left({ }^{\circ}\right)$ & $15.4 \pm 3.6$ & $11.0 \pm 0.9$ & $11.9 \pm 3.3$ \\
$\langle\alpha\rangle_{80}^{s}\left({ }^{\circ}\right)$ & $24.5 \pm 4.0$ & $22.6 \pm 0.8$ & $25.5 \pm 2.5$ \\
$\langle\alpha\rangle_{100}^{l}\left({ }^{\circ}\right)$ & $14.0 \pm 4.5$ & $12.8 \pm 1.0$ & $13.3 \pm 3.5$ \\
$\langle\alpha\rangle_{100}^{s}\left({ }^{\circ}\right)$ & $29.7 \pm 4.1$ & $27.8 \pm 0.9$ & $32.2 \pm 3.1$ \\
\hline
\end{tabular}

stable cases. There is a substantial misalignment $(\alpha \sim$ $10^{\circ}$ ) even at $40 \mathrm{~m}$ between the mean wind direction and the direction of the momentum flux. This should be expected since, from the Bolund measurements, we observed misalignment over the height range 5-12 m. The nonsmooth curves observed are a consequence of the low number of data points in the analysis, and hence an indicator of the statistical variation. The dependence of wind speed is not statistically significant, although we expect it to be present for the same reasons as described above for the angle $\gamma$.

\section{c. Vertical profiles of $\beta$}

The blue curves in Fig. 6 represent the angle $\beta$. For all three stability classes, it is close to zero at $40 \mathrm{~m}$, in agreement with the long fetch measurements from Bolund. They then decrease with height. Again, the largest misalignment is observed in the stable case. These findings directly contradict the assumptions inherent within the turbulent viscosity hypothesis in Eq. (1), which is the basis for most turbulent closure schemes used in surface-layer modeling.

The wind speed dependence is strong with increasing height for all the three stability classes and hence a more thorough investigation could have been made by refining the wind speed bins. The limited amount of data for this paper, however, made that impossible. The nonsmooth nature of the different profiles is due to the large statistical scatter that was also observed in Fig. $4 \mathrm{~b}$ for the neutral case for wind speeds between 8 and $10 \mathrm{~m} \mathrm{~s}^{-1}$.

\section{d. Large-eddy simulation}

Before drawing any conclusions, we will consider an ideal case from a large-eddy simulation (LES). We use data from high-resolution simulations generated using Sullivan and Patton's (2011) pseudospectral LES code. The code simulates the ABL over a flat, homogeneous terrain with high temporal and spatial resolution. A database containing LES data from this code has been
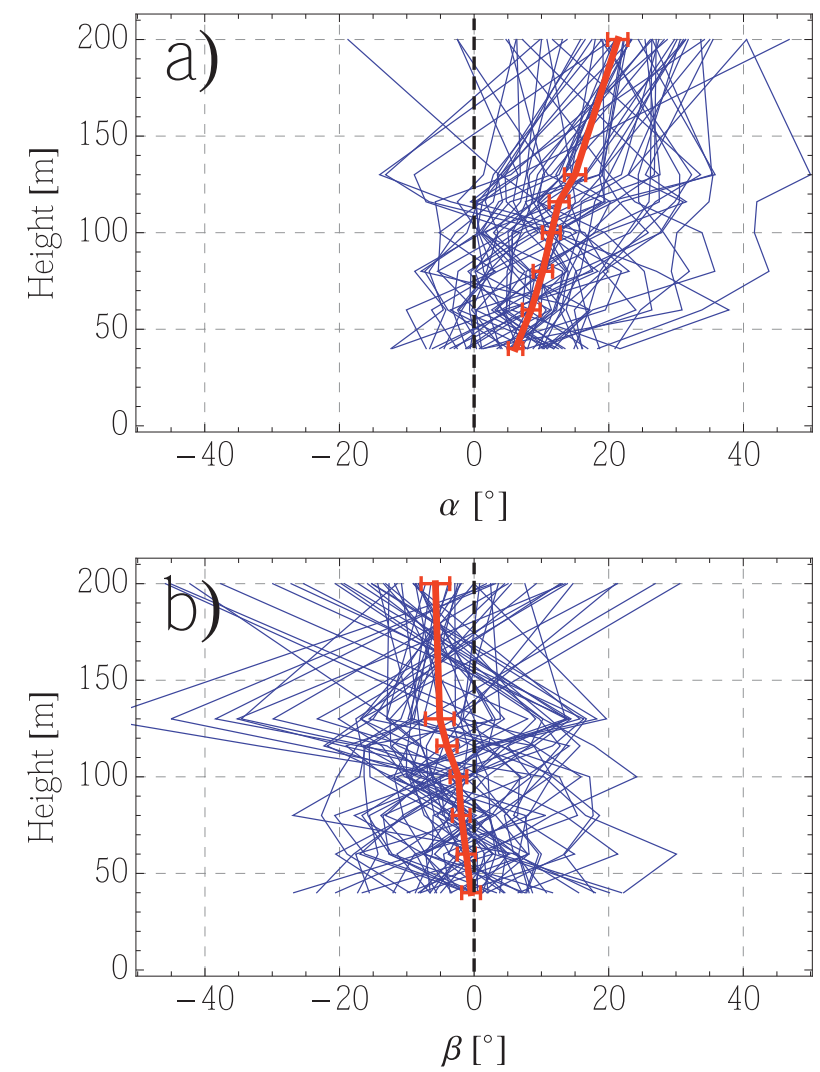

FIG. 5. Lidar measurements in neutral conditions of the angles (a) $\alpha$ and (b) $\beta$. Blue lines are the individual profiles for a 30-min sample (total number is 58), with red lines denoting associated standard error of the mean. Only profiles for which the 40-m wind speed is between 8 and $10 \mathrm{~m} \mathrm{~s}^{-1}$ are included.

established for different ABL states [for specific details on the LES database see Chougule et al. (2012)]. Since the stability classes and wind speeds are different from those measured at $\mathrm{H} \varnothing \mathbf{v} \varnothing \mathrm{re}$, we cannot expect exactly the same angle values. We, therefore, only focus on the qualitative differences.

The results of the LES are presented in Fig. 7. We have used the same color coding as in Fig. 6. In Fig. 7, $\alpha$ and $\gamma$ are in qualitative agreement with the WindCube data presented in Fig. 6 at Høvsøre: $\alpha$ grows much faster from the surface than $\gamma$, giving a significant positive value even at very shallow depths. On the other hand, $\beta$ does not display the negative values observed in Fig. 6 . In the LES it is very close to zero throughout the ABL for all three stability classes. In the stable case, $\beta$ turns negative at high altitudes. We attribute this to the $188-\mathrm{m}$ boundary layer height in the simulations.

\section{Discussion and conclusions}

The most striking finding in this paper is the misalignment between the stress vector and the vertical 

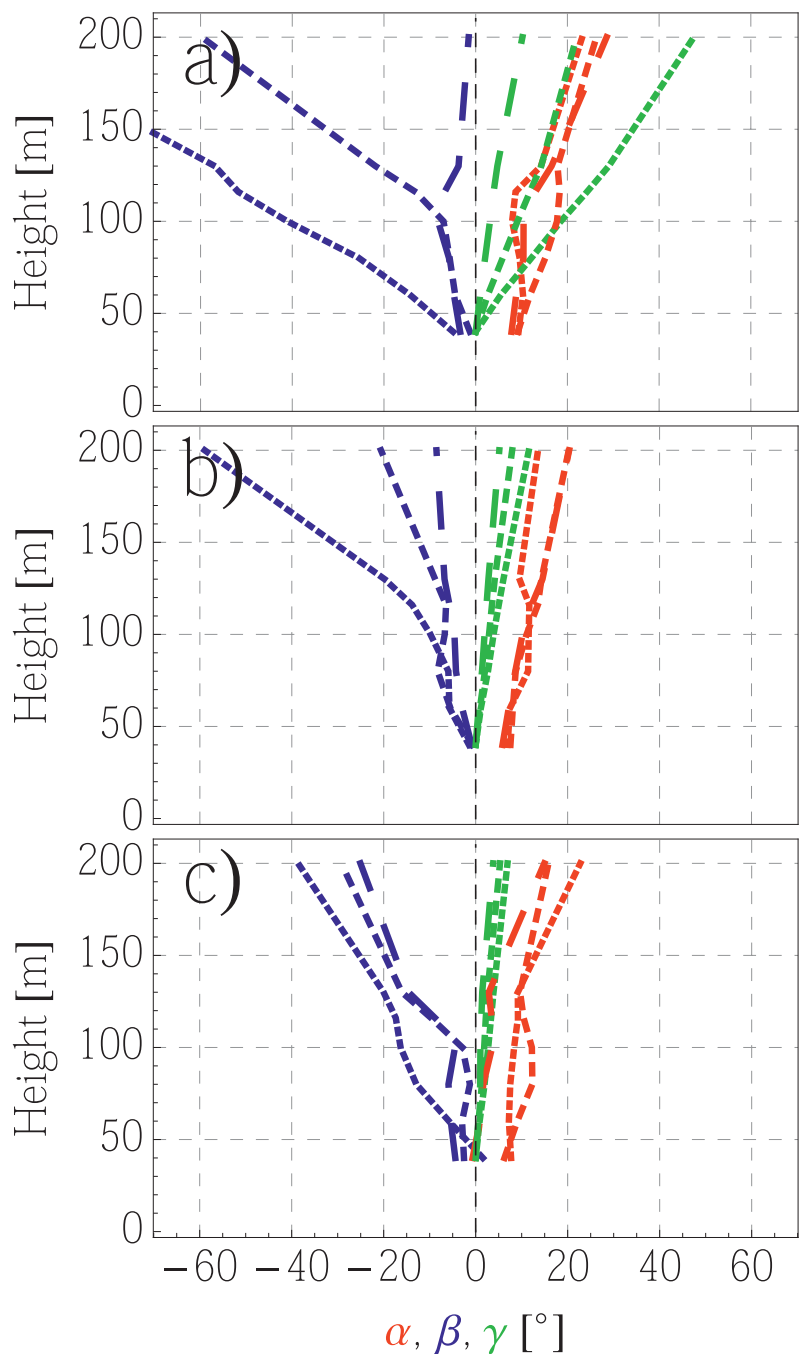

FIG. 6. Vertical profiles of $\alpha$ (red), $\beta$ (blue), and $\gamma$ (green) based on 30-min average samples in (a) stable, (b) neutral, and (c) unstable conditions. Line types represent different wind speed bins: 4-6 (dotted), 6-8 (short dashed), and 8-10 (long dashed) $\mathrm{m} \mathrm{s}^{-1}$. For stable conditions the total samples are 87 for low wind speed, and 17 and 9 for high wind speeds; for neutral, 44 for low wind speed, and 98 and 58 for high wind speeds; and for unstable, 53 for low wind speed, and 16 and 17 for high wind speeds.

gradient of the mean wind vector, resulting in the nonzero value of $\beta$ measured at Høvs $\varnothing$ re. In the LES we did not observe such negative values.

In flow over strictly homogenous and stationary surface conditions, it is difficult to think of situations in which $\beta$ is different from zero. Even small deviations from homogeneity at $\mathrm{H} \varnothing \mathrm{vs} \varnothing \mathrm{re}$ may, on the other hand, have significant impact on the measurements. According to Wyngaard (2010) the criterion for close to perfect surface scaling is $100-300$ times the measurement height. This is $10-30 \mathrm{~km}$ for measurements at $100 \mathrm{~m}$, which is very

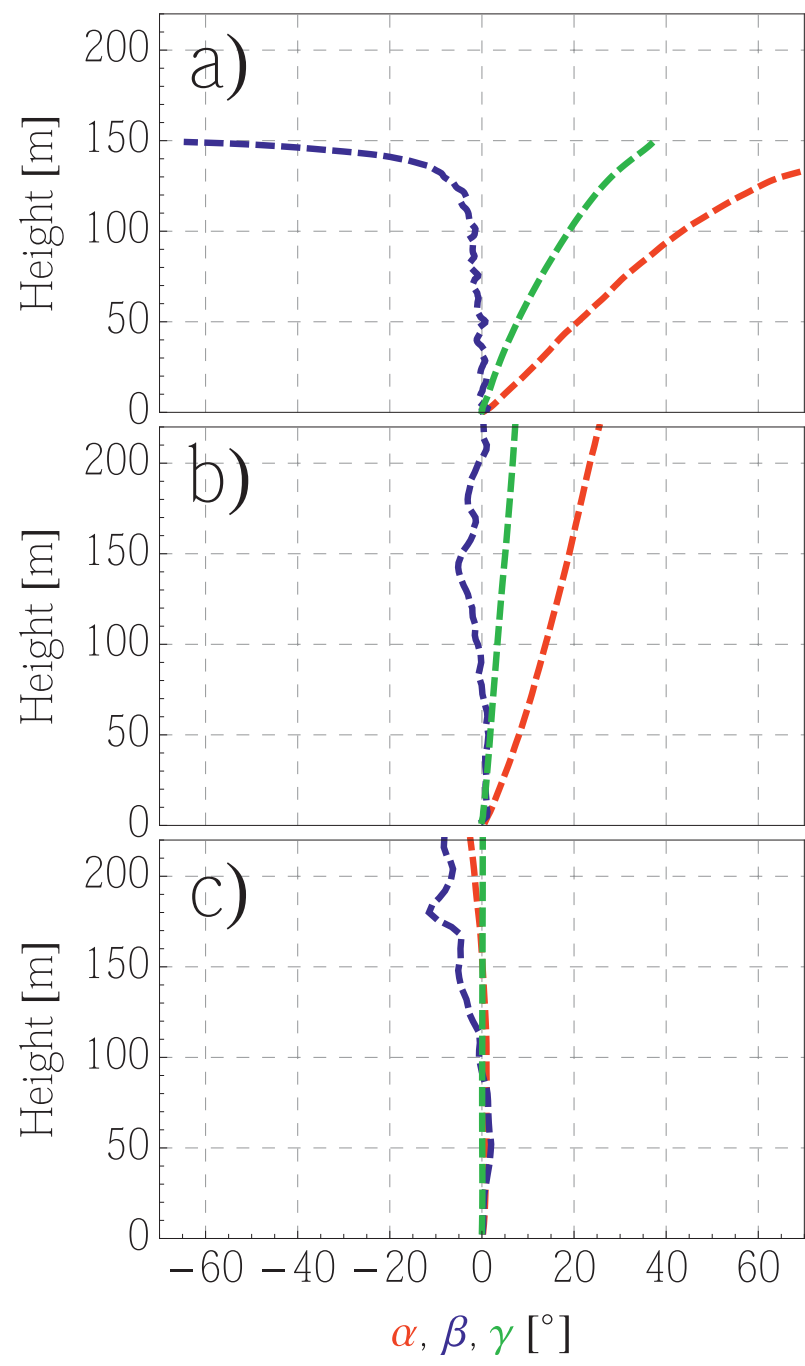

FIG. 7. LES vertical profiles of the angles $\alpha$ (red), $\beta$ (blue), and $\gamma$ (green) from LES for (a) stable, (b) neutral, and (c) unstable conditions.

difficult to obtain. In Fig. 3 we observe changes in surface roughness within $10 \mathrm{~km}$. With more data it would have been possible to select smaller wind direction bins in order to investigate this effect by avoiding critical sectors.

In general, we may need a theory that includes more than just local microscales in the turbulence description, for example, through a superposition of various long-fetch internal boundary layers. Determining whether baroclinic effects from horizontal temperature gradients are important in the description is also worth investigating. This could be achieved by considering profiles in different seasons. With the sea positioned only $2 \mathrm{~km}$ downstream of the site, there may be a potential impact. Recent studies show that under easterly winds, the average flow at Høvsøre is indeed baroclinic (R. Floors 2013, personal communication). 
To conclude, we have shown that the WindCube pulsed lidar is a useful tool for measuring horizontal angles of mean quantities in the ABL. We have also shown that the precise estimation of angles is accompanied by large scatter. This is mainly because of the difficulties in estimating the transverse component of the momentum flux vector. The sign, however, is well captured and agrees with a Northern Hemisphere atmospheric Ekman spiral. With an increasing number of lidars being utilized, we expect better statistics for future investigations.

Whether the findings, especially $\beta \neq 0$, have any practical consequences from a modeling viewpoint is yet to be determined. In LES the turbulent-viscosity hypothesis is only applied to the subgrid-scale model, and hence should not impose stringent controls on the resolved scales. Running LES with baroclinicity and roughness changes might provide new insight.

Acknowledgments. The work was partially supported by the Center for Computational Wind Turbine Aerodynamics and Atmospheric Turbulence under the Danish Council for Strategic Research, Grant 09-067216.

\section{REFERENCES}

Barnardes, M., and N. L. Dias, 2010: The alignment of the mean wind vector and stress vectors in the unstable surface layer. Bound.-Layer Meteor., 134, 41-59.

Berg, J., J. Mann, A. Bechmann, M. S. Courtney, and H. E. Jørgensen, 2011: The Bolund Experiment, part I: Flow over a steep, three-dimensional hill. Bound.-Layer Meteor., 141, 219-243.

Bradley, S., 2013: Aspects of the correlation between sodar and mast instrument winds. J. Atmos. Oceanic Technol., in press.

Chougule, A., J. Mann, M. Kelly, J. Sun, D. H. Lenschow, and E. G. Patton, 2012: Vertical cross-spectral phases in neutral atmospheric flow. J. Turbul., 13, doi:10.1080/14685248.2012.711524.

Clarke, R. H., and G. D. Hess, 1974: Geostrophic departure and the functions $A$ and $B$ of Rossby-number similarity theory. Bound.-Layer Meteor., 7, 267-287.

Eberhard, W. L., R. E. Cupp, and K. R. Healy, 1989: Doppler lidar measurement of profiles of turbulence and momentum flux. J. Atmos. Oceanic Technol., 6, 809-819.

Emeis, S., M. Harris, and R. M. Banta, 2007: Boundary-layer anemometry by optical remote sensing for wind energy applications. Meteor. Z., 16, 337-347, doi:10.1127/0941-2948/2007/ 0225 .

Frehlich, R., and L. Cornman, 2002: Estimating spatial velocity statistics with coherent Doppler lidar. J. Atmos. Oceanic Technol., 19, 355-366.
Gal-Chen, T., M. Xu, and W. L. Eberhard, 1992: Estimations of atmospheric boundary layer fluxes and other turbulence parameters from Doppler lidar data. J. Geophys. Res., 97 (D17), 18 409-18 423.

Grachev, A. A., C. W. Fairall, J. E. Hare, J. B. Edson, and S. D. Miller, 2003: Wind stress vector over ocean waves. J. Phys. Oceanogr., 33, 2408-2429.

Lothon, M., D. H. Lenschow, and S. D. Mayor, 2009: Doppler lidar measurements of vertical velocity spectra in the convective planetary boundary layer. Bound.-Layer Meteor., 132, 205226.

Mann, J., 1994: The spatial structure of neutral atmospheric surfacelayer turbulence. J. Fluid Mech., 273, 141-168.

- and Coauthors, 2009: Comparison of 3D turbulence measurements using three staring wind lidars and a sonic anemometer. Meteor. Z., 18, 135-140.

, A. Peña, F. Bingöl, R. Wagner, and M. S. Courtney, 2010: Lidar scanning of momentum flux in and above the atmospheric surface layer. J. Atmos. Oceanic Technol., 27, 959-976.

Mayor, S. D., D. H. Lenschow, R. L. Schwiesow, J. Mann, C. L. Frush, and M. K. Simon, 1997: Validation of NCAR 10.6- $\mu \mathrm{m}$ $\mathrm{CO}_{2}$ Doppler lidar radial velocity measurements and comparison with a 915-MHz profiler. J. Atmos. Oceanic Technol., 14, 1110-1126.

Peña, A., C. B. Hasager, S.-E. Gryning, M. Courtney, I. Antoniou, and T. Mikkelsen, 2009: Offshore wind profiling using light detection and ranging measurements. Wind Energy, 12, 105124.

— , S.-E. Gryning, J. Mann, and C. B. Hasager, 2010: Length scales of the neutral wind profile over homogeneous terrain. J. Appl. Meteor. Climatol., 49, 792-806.

Pichugina, Y. L., R. M. Banta, N. D. Kelley, B. J. Jonkman, S. C. Tucker, R. K. Newsom, and W. A. Brewer, 2008: Horizontalvelocity and variance measurements in the stable boundary layer using Doppler lidar: Sensitivity to averaging procedures. J. Atmos. Oceanic Technol., 25, 1307-1327.

Pope, S. B., 2000: Turbulent Flows. Cambridge University Press, $770 \mathrm{pp}$

Sathe, A., and J. Mann, 2012: Measurement of turbulence spectra using scanning pulsed wind lidars. J. Geophys. Res., 117, D01201, doi:10.1029/2011JD016786.

,-- J. Gottschall, and M. S. Courtney, 2011: Can wind lidars measure turbulence? J. Atmos. Oceanic Technol., 28, 853-868.

Sullivan, P. P., and E. G. Patton, 2011: The effect of mesh resolution on convective boundary layer statistics and structures generated by large-eddy simulation. J. Atmos. Sci., 68, 23952415.

Weber, R. O., 1997: Estimators for the standard deviation of horizontal wind direction. J. Appl. Meteor., 36, 1403-1415.

_ 1999: Remarks on the definition and estimation of friction velocity. Bound.-Layer Meteor., 93, 197-209.

Wyngaard, J. C., 2010: Turbulence in the Atmosphere. Cambridge University Press, 393 pp.

Yamartino, R. J., 1984: A comparison of several "single-pass" estimators of the standard deviation of wind direction. J. Appl. Meteor. Climatol., 23, 1362-1366. 